\title{
Technology for Submillimeter Astronomy
}

\author{
Jonas Zmuidzinas \\ Division of Physics, Mathematics, and Astronomy \\ California Institute of Technology, \\ Pasadena, CA 91125
}

\begin{abstract}
Despite about three decades of progress, the field of submillimeter astronomy remains quite challenging, because the detection technology is still under development and the transmission of the atmosphere is poor. The latter problem has been overcome by constructing submillimeter telescopes at excellent sites, first on Mauna Kea and later in Chile and Antarctica, and also by using airborne and space telescopes. Meanwhile, the improvements in technology over the past several decades have been remarkable. While considerable opportunities for improvement remain, existing detector and receiver technologies now often approach fundamental limits. This technological revolution has brought submillimeter astronomy from the fringes to the forefront of modern astrophysics and has stimulated major investments such as the 50-element ALMA interferometer and the ESA/NASA Herschel Space Observatory.
\end{abstract}

Index Terms-Terahertz, oscillators, detectors, mixers, superconducting, SIS, HEB, HEMT.

\section{INTRODUCTION}

The submillimeter band (loosely defined here as $300-$ $3000 \mathrm{GHz}$ ) represents a transition region between the coherent detection techniques used at radio wavelengths and the direct photon detection techniques used for infrared, optical, ultraviolet, and X-ray astronomy, and submillimeter astronomy has freely borrowed techniques from both regimes. As discussed in section II, there are both practical and fundamental reasons why the transition from coherent to direct detection occurs in the submillimeter. Here I provide a short introduction to the various technologies used for submillimeter astronomy; a number of review articles [1]-[17] may be consulted for more detail.

For coherent detection, the astronomical signal collected by the telescope is downconverted from the $\mathrm{THz}$ band to the $\mathrm{GHz}$ band over a limited bandwidth. The frequency conversion relies on a nonlinear element - the mixer - and a local oscillator. The output or intermediate-frequency (IF) signal can then be further processed using additional electronics, usually either a spectrometer or correlator. Alternatively, for direct detection the photons collected by the telescope are directly absorbed in some material, creating either electronic excitations or thermal energy (heat). In the latter case, the device is called a bolometer. In contrast to coherent receivers, spectral filtering must be accomplished prior to photon detection.

\section{Coherent vs. Direct Detection}

The primary distinction between coherent and incoherent (or direct) detection is the presence or absence of quantum noise. Coherent receivers and preserve information about both the amplitude and phase of the electromagnetic field while providing large photon number gain. As a result, coherent receivers are subject to quantum noise, which can be expressed as a minimum noise temperature of $T_{n}=h \nu / k_{B}$, or $48 \mathrm{~K} / \mathrm{THz}$ (see [18] and references therein). Quantum noise is equivalent to the shot noise produced by a background radiation flux of one photon per second per Hertz of detection bandwidth. At radio wavelengths, the background is significantly larger than this value and in any case never falls below the $2.7 \mathrm{~K}$ cosmic microwave background (CMB), and so the use of coherent receivers at radio wavelengths need not lead to a loss of sensitivity. In contrast, at optical or infrared wavelengths the quantum noise of coherent receivers is intolerably large, far larger than the typical backgrounds, and so direct detection is strongly preferred.

The parameter that best describes this transition is the photon occupation number $n$ of the background radiation, given by the Bose-Einstein formula $n=$ $\epsilon\left[e^{h \nu / k T}-1\right]^{-1}$ for an emissivity $\epsilon$ and temperature $T$. Coherent detection is competitive for $n \geq 1$, while direct detection is preferred when $n<<1$. For ground-based and airborne submillimeter telescopes, $n \geq 1$ [18], [19], while $n<<1$ is achievable in the submillimeter only with a cold space telescope [20]. This general rule for choosing between coherent and direct detection as a function of $n$ may be modified by practical considerations. For instance, continuum detection over wide bandwidths is generally easier using direct detection, while instrument size favors coherent detection for high-resolution spectroscopy [19].

In addition, one must also take into account the state of technology. When the first large submillimeter telescopes were built about 20 years ago, neither coherent receivers nor direct detectors performed particularly well in the submillimeter. Heterodyne mixers were either noisy or very narrow band. Local oscillators derived from electronics (using vaccum tubes or semiconductors) were very weak, while lasers were bulky, power hungry, and not continuously tunable, illustrating the so-called "THz gap" 
between electronic and optical technologies. Meanwhile direct detectors such as bolometers were difficult to manufacture even in small numbers, sensitive to microphonics, and were often not capable of reaching the background photon noise limit. As a result, the choice between coherent and direct detection was often dictated more by practical technological realities than by fundamental considerations such as quantum noise. This situation is now changing. As described in sections III and IV below, devices now often approach fundamental limits, and as a result the field is blossoming.

\section{Technology For Coherent Detection}

Because low-noise amplifiers are not available in the submillimeter band, the first operation that a coherent receiver must perform is frequency downconversion, or heterodyning, from $\mathrm{THz}$ to $\mathrm{GHz}$ frequencies. This is accomplished in the usual way, using a mixer and a local oscillator; however the mixer noise sets the system sensitivity and should be as low as possible.

Whisker-contacted GaAs Schottky diode mixers were commonly used in the early days of submillimeter astronomy [1], [8]. In addition to making electrical contact to the $\sim 1 \mu \mathrm{m}$ diode anode, the whisker also served as an antenna, either in a waveguide mount or above $\sim 500 \mathrm{GHz}$ in an open structure with a corner reflector (see [21] and references therein). For these higher frequencies the local oscillator power could only be provided by opticallypumped far-infrared gas lasers, which produced the necessary milliwatts of output power but operated only at fixed transition frequencies. Such receivers were the first to open up the short-wavelength end of submillimeter astronomy, initially at $690 \mathrm{GHz}[22]$ and eventually extending all the way to $5 \mathrm{THz}$ [23]. Developed somewhat earlier to about $600 \mathrm{GHz}$, receivers using $\mathrm{InSb}$ hot electron bolometer mixers [1], [24] and tunable diode-multiplied klystron oscillators were more sensitive, but had narrow $(\sim 1 \mathrm{MHz})$ bandwidths and required swept-frequency local oscillators in order to measure a spectrum.

The invention of the superconducting tunnel junction or "SIS" mixer [25], [26], the development of a detailed quantum theory of its operation [27], and the discovery of a method for producing robust, high quality tunnel junctions using the refractory metal niobium [28] have resulted in the development far more sensitive submillimeter mixers, often approaching the quantum limit [15], and extending up to frequencies as high as $1.25 \mathrm{THz}$ [29], [30]. The "photodiode picture" [10] provides the easiest way to understand the operation of SIS mixers. In this picture, each submillimeter photon absorbed causes one electron to tunnel across the junction barrier. Two conditions must generally be met in order to achieve quantum-limited sensitivity. First, a quantum efficiency approaching unity is needed which means that the impedance match between the tunnel junction resistance $R_{N}$ and the coupling circuit must be good. Consequently, the junction capacitance $C$ must be tuned out using a resonant circuit. since generally $\omega R_{N} C>1$. Second, to avoid excess noise, the photocurrent induced by the local oscillator must significantly exceed the subgap leakage current of the junction. In practice, the impedance matching is usually accomplished using on-chip superconducting circuitry along with careful electromagnetic design of the coupling structure. Meanwhile, low leakage currents are possible using modern junction fabrication technologies incorporating either aluminum oxide [28] or aluminum nitride [30], [31] barriers. SIS receivers have transformed submillimeter astronomy, and provided much of the motivation for pursuing largescale projects such as the ALMA interferometer [32] and the Herschel Space Observatory [29], [33].

Reverse photon assisted tunneling sets a hard upper frequency limit for SIS mixer operation. This limit is around $1.4 \mathrm{THz}$ for all-niobium junctions, and somewhat higher for junctions incorporating higher- $T_{c}$ materials, such as $\mathrm{Nb} / \mathrm{AlN} / \mathrm{NbTiN}$ [30]. At yet higher frequencies superconducting hot electron bolometer mixers [34], [35] now offer excellent performance (see [15] and references therein), and while quantum-limited detection remains elusive, their performance continues to improve as our understanding of their physics advances [36], [37].

The development of local oscillator technology has been equally impressive. Fueled by advances in computational electromagnetics, precision machining techniques, and especially device fabrication, frequency multipliers using GaAs diodes are now available to at least $2 \mathrm{THz}$ [38], [39]. These multipliers are driven either by phaselocked Gunn oscillators around $100 \mathrm{GHz}$, or in a more modern approach, by a microwave synthesizer multiplied up to $\sim 100 \mathrm{GHz}$. Above $2 \mathrm{THz}$, semiconductor quantum cascade lasers are showing great promise, and have already been demonstrated in conjunction with superconducting hot electron bolometer mixers [40], [41].

The active components are supported by the usual variety of passive devices familiar from microwave engineering, such as antennas or horns, transmission lines, waveguides, filters, couplers, hybrids, isolators, etc. However, these passives must usually operate at $\mathrm{THz}$ rather than $\mathrm{GHz}$ frequencies, and the mechanical fabrication of the corresponding waveguide structures becomes difficult. As a result, many of these functions are often implemented using free-space optical techniques. For instance, a beamsplitter serves as a directional coupler. For fabricating waveguide structures, standard metal machining remains dominant even above $1 \mathrm{THz}$ [39] although micromachining techniques have attracted interest [42], [43].

The IF output of a coherent receiver is sent to the "backend" electronics for spectroscopy or correlation. Although a variety of methods have been used for these functions, 
digital signal processing techniques are becoming increasingly dominant, especially using field programmable gate arrays (FPGA) [44]-[48].

\section{TEChNology For DiRECT DETECTION}

Direct detectors function by absorbing photons in a material and sensing the resulting change in a physical property of that material. In semiconductor photodetectors, the mobile charge carriers created by photons are sensed by measuring the current flow in response to an applied electric field. The minimum photon energy required to create an excitation sets a long-wavelength cutoff. One must therefore engineer semiconducting materials with appropriately small excitation energies in order to push detector operation from their native near-IR bands into the mid-IR and far-IR bands; successful approaches include the use of ternary alloys such as $\mathrm{Hg}_{x} \mathrm{Cd}_{1-x} \mathrm{Te}$, epitaxially grown quantum-well structures, and light doping with shallow impurities such as Ge:Ga. These methods become increasingly difficult at longer wavelengths and have not yet produced a successful submillimeter detector.

As a result, workers have focused instead on bolometers, in which the photon energy is directly converted to heat and then sensed using a thermistor. For a bolometer to be sensitive, one must have a very low thermal conductance $G$ between the "island" in which the photon energy is absorbed and the surrounding thermal bath. Indeed, $G$ sets the limiting sensitivity according to $\mathrm{NEP}^{2}=4 k T^{2} G$ [11]. In addition, the heat capacity $C$ of the island must be low, because the speed is limited by the thermal time constant $\tau=C / G$. Finally, the thermistor and its readout should not introduce excess noise; for example, the use of neutron transmutation doped (NTD) germanium thermistors along with silicon JFET preamplifiers provides a good solution.

Early devices were usually hand assembled "composite bolometers". The SCUBA instrument [49], which discovered submillimeter-bright galaxies at high redshifts, is a very successful example of this early technology. However, composite bolometer construction was very labor intensive and not amenable to scaling to large arrays: SCUBA used 37 pixels at $850 \mu \mathrm{m}$. This problem was overcome by the use of lithographic micromachining techniques, which in fact had been under development since the mid-1980's [50] but became widespread only starting in the mid1990's [51]-[53], enabling the production of arrays with up to a few hundred pixels.

The next step toward even larger arrays involved developing lithographically integrated thermistors and multiplexed readouts, such as superconducting transition-edge sensor (TES) thermistors [16], [54] coupled to timedomain SQUID multiplexing circuits [55]. As an example, 5000-pixel TES arrays are featured in the nearly completed SCUBA II [17], [56] instrument. Research in this area remains very vigorous. Alternative detection and multiplexing schemes are under active investigation [15], including superconducting photodetectors instead of bolometers, which should result in systems with even larger pixel counts using arrays that are relatively straightforward to fabricate [57]. Indeed, the availability of large arrays is providing strong motivation for the construction of a new large-aperture submillimeter telescope [20], [58].

\section{REFERENCES}

[1] T. Phillips and D. Woody, "Millimeter-wave and submillimeterwave receivers," Ann. Rev. Astron. Ap., vol. 20, pp. 285-321, 1982.

[2] J. Archer, "Low-noise heterodyne receivers for near-millimeter wave radio astronomy," Proc. IEEE, vol. 73, no. 1, pp. 109-130, 1985.

[3] J. R. Tucker and M. J. Feldman, "Quantum detection at millimeter wavelengths," Rev. Mod. Phys., vol. 57, no. 4, pp. 1055-1113, 1985.

[4] T. Phillips, "Submillimeter and far-infrared detectors," Astroph. Lett. Commun., vol. 26, no. 3-5, pp. 293-304, 1988.

[5] J. M. Payne, "Millimeter and submillimeter wavelength radio astronomy," Proc. IEEE, vol. 77, no. 7, pp. 993-1017, July 1989.

[6] P. Richards and Q. Hu, "Superconducting components for infrared and millimeter-wave receivers," Proc. IEEE, vol. 77, no. 8, pp. 1233-1246, 1989.

[7] R. Blundell and C.-Y. E. Tong, "Submillimeter receivers for radio astronomy," Proc. IEEE, vol. 80, no. 11, pp. 1702-1720, Nov. 1992.

[8] T. Crowe, R. Mattauch, H. Roser, W. Bishop, W. Peatman, and X. Liu, "GaAs Schottky diodes for THz mixing applications," Proc. IEEE, vol. 80, no. 11, pp. 1827-1841, 1992.

[9] T. G. Phillips and J. Keene, "Submillimeter astronomy," Proc. IEEE, vol. 80, pp. 1662-1678, Nov. 1992.

[10] M. J. Wengler, "Submillimeter-wave detection with superconducting tunnel diodes," Proc. IEEE, vol. 80, no. 11, pp. 1810-1826, Nov. 1992.

[11] P. Richards, "Bolometers for infrared and millimeter waves," $J$. Appl. Phys., vol. 76, no. 1, pp. 1-24, July 1994.

[12] J. Carlstrom and J. Zmuidzinas, "Millimeter and submillimeter techniques," in Review of Radio Science 1993-1996, W. R. Stone, Ed. Oxford University Press, June 1996, pp. 839-882.

[13] P. Siegel, "Terahertz technology," IEEE Trans. Microwave Theory Tech., vol. 50, no. 3, pp. 910-928, 2002.

[14] J. C. Webber and M. W. Pospieszalski, "Microwave instrumentation for radio astronomy," IEEE Trans. Microwave Theory Tech., vol. 50, no. 3, pp. 986-995, Mar. 2002.

[15] J. Zmuidzinas and P. L. Richards, "Superconducting detectors and mixers for millimeter and submillimeter astrophysics," Proc. IEEE, vol. 92, no. 10, pp. 1597-1616, Oct. 2004.

[16] K. D. Irwin and G. C. Hilton, "Transition-edge sensors," Top. Appl. Phys., vol. 99, pp. 63-149, 2005.

[17] K. D. Irwin, "Seeing with superconductors," Scientific American, vol. 295 , no. 5 , p. 86 , Nov. 2006.

[18] J. Zmuidzinas, "Thermal noise and correlations in photon detection," Appl. Optics, vol. 42, no. 25, pp. 4989-5008, Sept. 2003.

[19] — "The role of coherent detection," in New Concepts for FarInfrared and Submillimeter Space Astronomy, D. Benford and D. Leisawitz, Eds., vol. NASA/CP-2003-212233. Washington, DC: NASA, 2003, p. 329.

[20] C. Bradford and J. Zmuidzinas, "The future of far- $\mathbb{R}$ /submillimeter astrophysics with single-dish telescopes," in The Dense Interstellar Medium in Galaxies: Proceedings of the 4th Cologne-BonnZermatt Symposium, C. Kramer and J. Stutzki, Eds. Springer, 2004, p. 323.

[21] J. Zmuidzinas, A. L. Betz, and R. T. Boreiko, "A corner-reflector mixer mount for far infrared wavelengths," Infrared Physics, vol. 29, pp. 119-131, Jan. 1989.

[22] H. R. Fetterman, B. J. Clifton, D. D. Peck, P. E. Tannenwald, G. A. Koepf, P. F. Goldsmith, N. R. Erickson, D. Buhl, and N. McAvoy, "Submillimeter heterodyne detection of interstellar carbon monoxide at 434 micrometers," Science, vol. 211, pp. 580 582, Feb. 1981. 
[23] R. T. Boreiko and A. L. Betz, "Heterodyne Spectroscopy of the 63 Micron O I Line in M42," Ap. J. (Lett.), vol. 464, pp. L83-L86, June 1996.

[24] T. Phillips and K. Jefferts, "Low-temperature bolometer heterodyne receiver for millimeter wave astronomy," Rev. of Sci. Instrum., vol. 44, no. 8, pp. 1009-1014, 1973.

[25] G. J. Dolan, T. G. Phillips, and D. P. Woody, "Low-noise 115-GHz mixing in superconducting oxide-barrier tunnel junctions," Appl. Phys. Lett., vol. 34, no. 5, pp. 347-349, Mar. 1979.

[26] P. L. Richards, T. M. Shen, R. E. Harris, and F. L. Lloyd, "Quasiparticle heterodyne mixing in SIS tunnel junctions," Appl. Phys. Lett., vol. 34, no. 5, pp. 345-347, Mar. 1979.

[27] J. Tucker, "Quantum limited detection in tunnel junction mixers," IEEE J. Quant. Electron., vol. 15, no. 11, pp. 1234-1258, 1979.

[28] M. Gurvitch, M. A. Washington, and H. A. Huggins, "High quality refractory Josephson tunnel junctions utilizing thin aluminum layers," Appl. Phys. Lett., vol. 42, pp. 472-474, Mar. 1983.

[29] B. D. Jackson, G. de Lange, T. Zijlstra, M. Kroug, J. W. Kooi, J. A. Stern, and T. M. Klapwijk, "Low-noise $0.8-0.96$ and $0.96-1.12$ thz superconductor-insulator-superconductor mixers for the Herschel Space Observatory," IEEE Trans. Microwave Theory Tech., vol. 54, no. 2, p. $547,2006$.

[30] A. Karpov, D. Miller, F. Rice, J. A. Stern, B. Bumble, H. G. LeDuc, and J. Zmuidzinas, "Development of $1.25 \mathrm{THz}$ SIS mixer for Herschel Space Observatory," in Millimeter and Submillimeter Detectors and Instrumentation for Astronomy III, J. Zmuidzinas, W. S. Holland, S. Withington, and W. D. Duncan, Eds. Proc. SPIE, vol. 6275,2006 , pp. 62751U-1 - 62751U-6.

[31] J. Kawamura, D. Miller, J. Chen, J. Zmuidzinas, B. Bumble, H. G. LeDuc, and J. A. Stern, "Very high current density Nb/AlN/Nb tunnel junctions for low-noise submillimeter mixers," Appl. Phys. Lett., vol. 76, no. 15, pp. 2119-2121, Apr. 2000.

[32] A. Wooten, "Atacama large millimeter array (ALMA)," in Large Ground-based Telescopes, J. M. Oschmann and L. M. Stepp, Eds., vol. 4837. Proc. SPIE, vol. 4837, Feb. 2003, pp. 110-118.

[33] G. L. Pilbratt, "Herschel Space Observatory mission overview," in IR Space Telescopes and Instruments, J. C. Mather, Ed. Proc. SPIE, vol. 4850, Mar. 2003, pp. 586-597.

[34] E. Gershenzon, G. Gol'tsman, Y. Gousev, A. Elantev, and A. Semenov, "Electromagnetic-radiation mixer based on electron heating in resistive state of superconductive $\mathrm{Nb}$ and $\mathrm{YBaCuO}$ films," IEEE Trans. Magnetics, vol. 27, no. 2, pp. 1317-1320, 1991.

[35] D. Prober, "Superconducting terahertz mixer using a transitionedge microbolometer," Appl. Phys. Lett., vol. 62, no. 17, pp. 2119 2121,1993

[36] M. Hajenius, J. Baselmans, A. Baryshev, J. R. Gao, T. M. Klapwijk, J. W. Kooi, W. Jellema, and Z. Q. Yang, "Full characterization and analysis of a terahertz heterodyne receiver based on a $\mathrm{NbN}$ hot electron bolometer," J. Appl. Phys., vol. 100, p. art. \#074507, Oct. 2006.

[37] E. L. Kollberg and K. S. Yngvesson, "Quantum-noise theory for terahertz hot electron bolometers," IEEE Trans. Microwave Theory Tech., vol. 54, no. 5, pp. 2077-2089, May 2006.

[38] I. Mehdi, E. Schlecht, G. Chattopadhyay, and P. H. Siegel, "THz local oscillator sources," in Proceedings far-IR, $s u b-\mathrm{mm}$, and $\mathrm{mm}$ detector workshop, J. Wolf, J. Farhoomand, and C. R. McCreight, Eds., vol. NASA/CP-2003-211408. Washington, DC: NASA, 2003, pp. 161-166.

[39] A. Maestrini, J. S. Ward, H. Javadi, C. Tripon-Canseliet, J. Gill, G. Chattopadhyay, E. Schlecht, and I. Mehdi, "Local oscillator chain for 1.55 to $1.75 \mathrm{THz}$ with $100 \mu \mathrm{W}$ peak power," IEEE Micr. Wireless Comp. Lett., vol. 15, pp. 871-873, Dec. 2005.

[40] J. R. Gao, J. N. Hovenier, Z. Q. Yang, J. A. Baselmans, A. Baryshev, M. Hajenius, T. M. Klapwijk, A. J. L. Adam, T. O. Klaassen, B. S. Williams, S. Kumar, Q. Hu, and K. L. Reno, "Terahertz heterodyne receiver based on a quantum cascade laser and a superconducting bolometer," Appl. Phys. Lett., vol. 86, no. 24, p. art. \#244104, June 2005.

[41] H. W. Hübers, S. G. Pavlov, H. Richter, A. D. Semenov, L. Mahler, A. Treducci, H. E. Beere, and D. A. Ritchie, "High-resolution gas phase spectroscopy with a distributed feedback terahertz quantum cascade laser," Appl. Phys. Lett., vol. 89, no. 6, p. art. $\# 061115$, Aug. 2006.

[42] V. M. Lubecke, K. Mizuno, and G. M. Rebeiz, "Micromachining for terahertz applications," IEEE Trans. Microwave Theory Tech. vol. 46, no. 11, pp. 1821-1831, Nov. 1998.

[43] P. L. Kirby, D. Pukala, H. Manohara, I. Mehdi, and J. Papapolymerou, "Characterization of micromachined silicon rectangular waveguide at $400 \mathrm{GHz}$," IEEE Micr. Wireless Comp. Lett., vol. 16, no. 6, pp. 366-368, June 2006.

[44] B. von Herzen, "Digital cross-correlation at $250 \mathrm{MHz}$ using highperformance FPGAs," in Advanced Technology MMW, Radio, and Terahertz Telescopes, T. G. Phillips, Ed. Proc. SPIE, vol. 3357, July 1998 , pp. $395-403$.

[45] D. W. Hawkins, D. P. Woody, B. Wiitala, J. Fredsti, and K. P. Rauch, "The CARMA correlator," in Millimeter and Submillimeter Detectors for Astronomy II, J. Zmuidzinas, W. S. Holland, and S. Withington, Eds. Proc. SPIE, vol. 5498, 2004, pp. 567-578.

[46] A. O. Benz, P. C. Grigis, V. Hungerbuihler, H. Meyer, C. Monstein, B. Stuber, and D. Zardet, "A broadband FFT spectrometer for radio and millimeter astronomy," Astron. Ap., vol. 442, pp. 767-773, Nov. 2005.

[47] B. Klein, S. D. Philipp, I. Krämer, C. Kasemann, R. Guisten, and K. M. Menten, "The APEX digital Fast Fourier Transform Spectrometer," Astron. Ap., vol. 454, pp. L29-L32, Aug. 2006.

[48] D. Werthimer, "FPGA based spectrometers, beam formers, and correlators," 2006, unpublished; see http://ral.berkeley.edu/ata/Publications/werthimer_ursi2006.ppt.

[49] W. Holland, E. Robson, W. Gear, C. Cunningham, J. Lightfoot, T. Jenness, R. Ivison, J. Stevens, P. Ade, M. Griffin, W. Duncan, J. Murphy, and D. Naylor, "SCUBA: a common-user submillimetre camera operating on the James Clerk Maxwell Telescope," Mon. Not. Roy. Astron. Soc., vol. 303, no. 4, pp. 659-672, 1999.

[50] S. Moseley, J. Mather, and D. McCammon, "Thermal detectors as X-ray spectrometers," J. Appl. Phys., vol. 56, no. 5, pp. 1257-1262, 1984.

[51] J. J. Bock, D. Chen, P. D. Mauskopf, and A. E. Lange, "A novel bolometer for infrared and millimeter-wave astrophysics," Space Science Reviews, vol. 74, pp. 229-235, Oct. 1995.

[52] E. Kreysa, J. W. Beeman, and E. E. Haller, "Micromachined bolometer arrays with silicon nitride membranes," in Proc. 30th ESLAB Symp.: Submillimetre and far-infrared space instrumentation, vol. ESA SP-388, 1996.

[53] S. H. Moseley, C. A. Allen, D. Benford, C. D. Dowell, D. A. Harper, T. G. Phillips, R. F. Silverberg, and J. Staguhn, "The design and performance of the 384-element imaging submillimeter detector arrays for HAWC and SHARC II," Nucl. Instrum. Meth. Phys. A, vol. 520, pp. 417-420, Mar. 2004

[54] K. Irwin, "An application of electrothermal feedback for highresolution cryogenic particle-detection," Appl. Phys. Lett., vol. 66, no. 15 , pp. 1998-2000, 1995.

[55] J. Chervenak, K. Irwin, E. Grossman, J. Martinis, C. Reintsema, and M. Huber, "Superconducting multiplexer for arrays of transition edge sensors," Appl. Phys. Lett., vol. 74, no. 26, pp. 4043-4045, 1999.

[56] W. S. Holland, W. Duncan, B. D. Kelly, K. D. Irwin, A. J. Walton, P. A. R. Ade, and E. I. Robson, "SCUBA-2: a new generation submillimeter imager for the James Clerk Maxwell Telescope," in Millimeter and Submillimeter Detectors for Astronomy, T. G. Phillips and J. Zmuidzinas, Eds. Proc. SPIE, vol. 4855, Feb. 2003, pp. 1-18.

[57] P. Day, H. LeDuc, B. Mazin, A. Vayonakis, and J. Zmuidzinas, "A broadband superconducting detector suitable for use in large arrays," Nature, vol. 425, no. 6960, pp. 817-821, 2003.

[58] T. A. Sebring, R. Giovanelli, S. Radford, and J. Zmuidzinas, "Cornell Caltech Atacama Telescope (CCAT): a 25-m aperture telescope above 5000-m altitude," in Ground-based and Airborne Telescopes, L. M. Stepp, Ed. Proc. SPIE, Vol. 6267, 2006, p. $62672 \mathrm{C}$. 